\title{
Performance of Fenugreek (Trigonella foenum-graecum L.) Varieties for Table Purpose under Kharif Season
}

\author{
Supriya K. Ghadge*, A. A. Shaikh, J. D. Jadhav, V. A. Sthool, \\ A. B. Bhosale and S. V. Bagade \\ Department of Agricultural Meteorology, College of Agriculture, Pune, Mahatma Phule \\ Krishi Vidyapeeth Rahuri Maharashtra State, India \\ *Corresponding author
}

Keywords

Fenugreek, Variety, Yield

Article Info

Accepted:

20 January 2021

Available Online:

10 February 2021
An experiment was carried out at Department of Agricultural Meteorology Research Farm, College of Agriculture, and Pune during kharif, 2019. Field experiment was laid out in a split plot design with three replications. There were sixteen treatment combinations comprising of four varieties (Phule Kasturi, RMT-1, Methi Extra Bold and Local Variety) as main plot treatment and four sowing windows $(26,27,28$ and $29 \mathrm{MW})$ as sub plot treatment. The gross and net plot sizes were $3.0 \mathrm{~m} \times 2.0 \mathrm{~m}$ and $2.6 \mathrm{~m} \times 1.8 \mathrm{~m}$, respectively. The plant protection measures were given as per requirement. The periodical observations on growth and yield contributing characters were recorded to assess the treatment effects. Different varieties of fenugreek showed a significant influence on growth and yield parameters. Among the four cultivars of table purpose fenugreek, Phule Kasturi recorded significantly higher growth components like plant height, number of functional leaves plant $^{-1}$, leaf area plant ${ }^{-1}$, dry matter accumulation plant ${ }^{-1}$ and yield components resulted in increased fresh foliage yield as compared to RMT-1, Methi Extra Bold and Local Variety. From this investigation, it was concluded that table purpose fenugreek variety Phule Kasturi was found good and optimum time for sowing of table purpose fenugreek should be last week of June. A table purpose fenugreek variety Phule Kasturi when sown during 26 MW (25th June ${ }^{-1}$ st July) favorably influenced growth and yield contributing characters resulted in better yields.

\section{Introduction}

Fenugreek (Trigonella foenum-graecum L.) belonging to the family fabaceae, is an important leafy vegetable as well as an important seed spice. Because of its various uses, fenugreek ranks as the third most important seed spice of India after coriander and cumin. Fenugreek is a multipurpose crop being used as a leafy vegetable, spice, fodder and medicinal plant. The fresh tender leaves and shoots are much used as a vegetable for human consumption since ancient times and as forage for cattle and also in medicine (Govindaraj et al., 2019). The name fenugreek came from the species name "foenum-graecum" means "Greek hay" (Flammang et al., 2004). It is an annual herb having light green leaves, pinnately trifoliate, flowers papilionaceous, long, narrow, curved, 
tapering with a slender point and containing small deeply furrowed seeds. There are two species of the genus Trigonella, which are of economic importance viz., T. foenum graecum, the common methi and $T$. corniculata, the Kasuri methi. These two differ in their growth habit and yield. The latter one is a slow growing type and remains in rosette condition during most of its vegetative growth period.

The leaves and fruits have a pleasant aromatic odour. Fresh fenugreek leaves as part of the fenugreek plant is used as an edible herb. Now a days, the consumption of leaves has considerably gained attention in stabilizing the insulin, blood sugar, haemoglobin levels and condition of diabetes (Mehta et al., 2013).Fenugreek contains 23 to $26 \%$ protein, 6 to $7 \%$ fat and $58 \%$ carbohydrates of which about $25 \%$ is dietary fiber (US Department of Agriculture, 2012).

Performance of the fenugreek crop varies from variety to variety and season to season due to variation in agroecological conditions. With this consideration in view, the investigation was planned with the objective to find out suitable variety of fenugreek under kharif season.

\section{Materials and Methods}

A field experiment was conducted on growth and yield parameters of fenugreek at Department of Agricultural Meteorology Research Farm, College of Agriculture, Pune during kharif, 2019. A total of four varieties viz., Phule Kasturi, RMT-1, Methi Extra Bold and Local Variety were evaluated on four sowing dates at 7- days interval (26 MW, 27 MW, $28 \mathrm{MW}$ and $29 \mathrm{MW}$ ) in split plot design with four main plots as varieties and four subplots as sowing dates. Recommended practices were followed. All the observations on growth parameters were recorded at the different growth stages of plant and observations on yield of fenugreek were recorded after harvesting of the crop. Details of treatments along with their symbols are mentioned in Table 1.

\section{Results and Discussion}

The results obtained from different growth characters viz., plant count, plant height, number of functional leaves, dry matter accumulation plant ${ }^{-1}$, leaf area plant ${ }^{-1}$ and yield character like fresh foliage yield plant $^{-1}$ and $\mathrm{ha}^{-1}$ were studied. The experimental results are interpreted along with the corresponding tables and figures 1-10.

\section{Plant count}

\section{Initial and final plant count}

The initial plant count in fenugreek was recorded ten days after each sowing date, whereas, final plant count was recorded at harvest.

The mean initial plant count after ten days of sowing $\left(\mathrm{ha}^{-1}\right)$ and its percentage was found 33.26 lakh ha ${ }^{-1}$ and $96.90 \%$, respectively, whereas, the final plant count at harvest $\left(\mathrm{ha}^{-1}\right)$ with its percentage were 33.18 lakh ha ${ }^{-1}$ and $96.61 \%$, respectively (Table 2, Fig.1).

The mean initial and final plant counts $\left(\mathrm{ha}^{-1}\right)$ in fenugreek and their percentage were not significantly influenced by different varieties of fenugreek indicating that variation obtained in the investigation is the mere differences due to treatments tried.

\section{Growth characters}

\section{Plant height}

Data on mean plant height of fenugreek as influenced periodically are presented in Table 
3 and graphically depicted in Fig.2. It showed that the mean plant height progressively increased up to 21 DAS and then its growth rate gradually slowed down and reached up to $18.78 \mathrm{~cm}$ at harvest. The mean plant height at $7,14,21,28$ DAS and at harvest was found to be $3.37 \mathrm{~cm}, 8.25 \mathrm{~cm}, 12.84 \mathrm{~cm}, 17.20 \mathrm{~cm}$ and $18.78 \mathrm{~cm}$, respectively.

The plant height of fenugreek was significantly influenced by varieties at all the stages of crop growth. A variety Phule Kasturi was found significantly superior over other varieties in producing taller plants up to harvest. The mean plant height of Phule Kasturi was significantly superior at 7, 14, 21, 28 DAS and at harvest i.e. 4.04, 8.77, 13.61, 18.20 and $19.67 \mathrm{~cm}$, respectively, over varieties RMT-1, Methi extra bold and Local variety during all the growth stages (Table 7). It might be due to its genetical character of variety Phule Kasturi. Revanappa et al., (2012), Aggrwal et al., (2013), Singh et al., (2013), Sharma (2015), Girdhar et al., (2016) and Latye et al., (2016) reported similar findings.

\section{Mean number of functional leaves plant ${ }^{-1}$}

The data recorded on mean number of functional leaves plant $^{-1}$ in fenugreek as affected periodically by different treatments are presented in Table 4 and graphically represented in Fig.3. Data revealed that the number of functional leaves increased from 2.10 at 7 days to 16.53 at harvest.

The difference between the mean number of functional leaves plant ${ }^{-1}$ in different fenugreek varieties showed significant results at all the crop growth stages (Table 4). The highest number of functional leaves recorded (18.07) with variety Phule Kasturi at harvest. This might be due to genetical character of variety Phule Kasturi. Revanappa et al., (2012), Aggrwal et al., (2013), Singh et al., (2013),
Sharma (2015), Girdhar et al., (2016) and Latye et al., (2016) reported similar findings.

\section{Leaf area plant ${ }^{-1}\left(\mathrm{~cm}^{2}\right)$}

The data recorded on mean leaf area plant ${ }^{-1}$ in fenugreek as affected by different treatments are presented in Table 5 and graphically depicted in Fig.4.

The mean leaf area plant $^{-1}$ was $1.59,5.17$, $13.69,22.50$ and $23.94 \mathrm{~cm} 2$ recorded at 7, 14, 21,28 days after sowing and at harvest, respectively. It was observed that the mean leaf area increased up to harvest.

The mean leaf area plant ${ }^{-1}$ was significantly affected due to different fenugreek varieties at $7,14,21,28$ days after sowing and at harvest. A variety Phule Kasturi recorded higher leaf area $(24.69 \mathrm{~cm} 2)$ than rest of the varieties at harvest. This might be due to genetical characters of Phule Kasturi. Revanappa et al., (2012), Aggrwal et al., (2013), Singh et al., (2013), Sharma (2015), Girdhar et al., (2016) and Latye et al., (2016) reported similar findings.

\section{Dry matter plant ${ }^{-1}$}

Data in respect of periodical accumulation of mean total dry matter plant ${ }^{-1}(\mathrm{mg})$ amongst the various plant parts of fenugreek as affected by various treatments are presented in Table 6 and graphically presented in Fig.5. Mean dry matter plant ${ }^{-1}$ at 7, 14, 21, 28 DAS and at harvest was $74.00,151.91,312.41$, 386.83 and $442.31 \mathrm{mg} \mathrm{plant}^{-1}$, respectively. At initial stages, the rate of increase of dry matter was the highest between 7 to 21 DAS. Thereafter, the rate of increase of dry matter gradually slowed down.

Mean total dry matter accumulation plant ${ }^{-1}$ was significantly influenced due to fenugreek varieties at all the stages of crop growth 
(Table 6). A variety Phule Kasturi significantly produced maximum dry matter plant $^{-1} 87.75,169.99,338.93,413.98$ and $476.45 \mathrm{mg}$ at $7,14,21,28$ and at harvest, respectively, over variety Methi Extra Bold, RMT-1 and Local variety. This might be due to genetical characters of Phule Kasturi. Revanappa et al., (2012), Aggrwal et al., (2013), Singh et al., (2013), Sharma (2015), Girdhar et al., (2016) and Latye et al., (2016) reported similar findings.

Table.1 Details of treatments along with their symbols

\begin{tabular}{|l|l|l|l|}
\hline \multicolumn{2}{|l|}{ A) Main plot : Varieties (4) } & \multicolumn{2}{l|}{ B) Sub plot: Sowing Windows (4) } \\
\hline$V_{\mathbf{1}}$ & PhuleKasturi & $\mathrm{S}_{1}$ & MW 26 (25 June-1 July) \\
\hline$V_{\mathbf{2}}$ & RMT-1 & $\mathrm{S}_{2}$ & MW 27 (2 July-8 July) \\
\hline$V_{\mathbf{3}}$ & Methi Extra Bold & $\mathrm{S}_{3}$ & MW 28 (9 July-15 July) \\
\hline$V_{\mathbf{4}}$ & Local Variety & S $_{\mathbf{4}}$ & MW 29 (16 July-22 July) \\
\hline
\end{tabular}

Fig.1 Mean initial and final plant count $\left(\mathrm{ha}^{-1}\right)$ as affected by different varieties in fenugreek

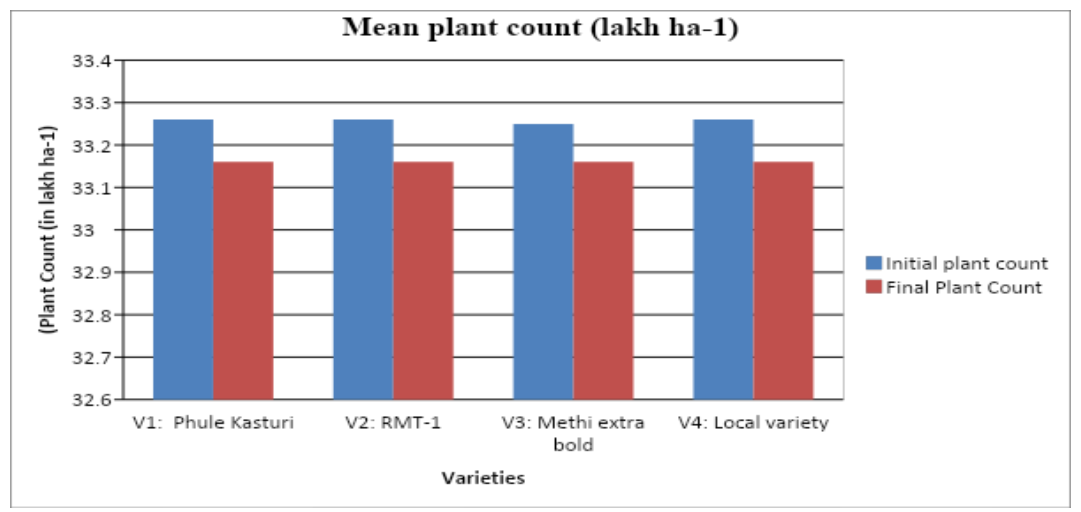


Table.2 Mean plant population as affected by different treatments in fenugreek

\begin{tabular}{|c|c|c|c|c|}
\hline \multirow{3}{*}{ Treatment } & \multicolumn{4}{|c|}{ Plant count } \\
\hline & \multicolumn{2}{|c|}{ Initial } & \multicolumn{2}{|c|}{ Final } \\
\hline & $\left(\right.$ lakh ha $\left.{ }^{-1}\right)$ & (\%) to total & (lakh ha ${ }^{-1}$ ) & (\%) to total \\
\hline \multicolumn{5}{|l|}{ Varieties (V) } \\
\hline 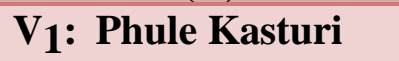 & 33.26 & 96.91 & 33.16 & 96.61 \\
\hline V2: RMT-1 $_{2}$ & 33.26 & 96.90 & 33.16 & 96.61 \\
\hline V3: Methi extra bold & 33.25 & 96.89 & 33.16 & 96.61 \\
\hline V4: Local variety & 33.26 & 96.91 & 33.16 & 96.61 \\
\hline S.Em \pm & 0.01 & 0.02 & 0.01 & 0.02 \\
\hline C.D. at $5 \%$ & NS & NS & NS & NS \\
\hline General mean & 33.26 & 96.90 & 33.18 & 96.61 \\
\hline
\end{tabular}

Table.3 Mean plant height $(\mathrm{cm})$ as affected periodically by different treatments in Fenugreek

\begin{tabular}{|l|c|c|c|c|c|}
\hline & \multicolumn{5}{|c|}{ Davs after sowing } \\
\cline { 2 - 6 } & $\mathbf{7}$ & $\mathbf{1 4}$ & $\mathbf{2 1}$ & $\mathbf{2 8}$ & At \\
\hline Varieties (V) & 4.04 & 8.77 & 13.61 & 18.20 & $\mathbf{1 9 . 6 7}$ \\
\hline V $_{\mathbf{1}}$ Phule Kasturi & 3.74 & 8.64 & 13.46 & 17.62 & $\mathbf{1 9 . 4 5}$ \\
\hline V2: RMT-1 & 3.18 & 8.09 & 12.90 & 17.27 & $\mathbf{1 8 . 7 7}$ \\
\hline V3: Methi Extra Bold & 2.52 & 7.49 & 11.39 & 15.73 & $\mathbf{1 7 . 2 3}$ \\
\hline V4 : Local variety & 0.02 & 0.03 & 0.02 & 0.02 & $\mathbf{0 . 0 2}$ \\
\hline S.Em \pm & 0.08 & 0.10 & 0.05 & 0.07 & $\mathbf{0 . 0 6}$ \\
\hline C.D. at 5 \% & $\mathbf{3 . 3 7}$ & $\mathbf{8 . 2 5}$ & $\mathbf{1 2 . 8 4}$ & $\mathbf{1 7 . 2 0}$ & $\mathbf{1 8 . 7 8}$ \\
\hline General mean & & & & & \\
\hline
\end{tabular}

Table.4 Mean number of functional leaves plant ${ }^{-1}$ as affected periodically by different treatments in fenugreek

\begin{tabular}{|l|c|c|c|c|c|}
\hline \multirow{2}{*}{ Treatment } & \multicolumn{5}{|c|}{ Days after sowing } \\
\cline { 2 - 6 } & $\mathbf{7}$ & $\mathbf{1 4}$ & $\mathbf{2 1}$ & $\mathbf{2 8}$ & At harvest \\
\hline Varieties (V) & & & & & \\
\hline V1: Phule Kasturi & 2.28 & 6.23 & 9.18 & 15.03 & $\mathbf{1 8 . 0 7}$ \\
\hline V2: RMT-1 & 2.00 & 5.83 & 8.58 & 14.33 & $\mathbf{1 5 . 9 5}$ \\
\hline V3: Methi Extra Bold & 2.12 & 6.07 & 8.90 & 14.80 & $\mathbf{1 6 . 8 4}$ \\
\hline V4 : Local variety & 2.00 & 5.17 & 7.99 & 13.98 & $\mathbf{1 5 . 2 7}$ \\
\hline S.Em & 0.02 & 0.05 & 0.04 & 0.05 & $\mathbf{0 . 0 6}$ \\
\hline C.D. at 5 \% & 0.07 & 0.18 & 0.12 & 0.19 & $\mathbf{0 . 2 0}$ \\
\hline General mean & $\mathbf{2 . 1 0}$ & $\mathbf{6 . 8 3}$ & $\mathbf{8 . 6 6}$ & $\mathbf{1 4 . 5 4}$ & $\mathbf{1 6 . 5 3}$ \\
\hline
\end{tabular}


Table.5 Mean leaf area plant ${ }^{-1}\left(\mathrm{~cm}^{2}\right)$ as affected periodically by different treatments in fenugreek

\begin{tabular}{|l|c|c|c|c|c|}
\hline \multirow{2}{*}{ Treatment } & \multicolumn{5}{|c|}{ Days after sowing } \\
\cline { 2 - 6 } & $\mathbf{7}$ & $\mathbf{1 4}$ & $\mathbf{2 1}$ & $\mathbf{2 8}$ & At \\
\hline Varieties (V) & & & & \\
\hline V1: Phule Kasturi & 1.68 & 5.39 & 14.66 & 23.47 & $\mathbf{2 4 . 6 9}$ \\
\hline V2: RMT-1 & 1.57 & 5.20 & 14.02 & 22.61 & $\mathbf{2 4 . 1 0}$ \\
\hline V3: Methi Extra Bold & 1.64 & 5.29 & 14.47 & 22.89 & $\mathbf{2 4 . 4 0}$ \\
\hline V4 : Local variety & 1.48 & 4.79 & 11.61 & 21.04 & $\mathbf{2 2 . 5 7}$ \\
\hline S.Em+ & 0.03 & 0.01 & 0.03 & 0.07 & $\mathbf{0 . 0 2}$ \\
\hline C.D. at 5 \% & 0.11 & 0.05 & 0.09 & 0.24 & $\mathbf{0 . 0 7}$ \\
\hline General mean & $\mathbf{1 . 5 9}$ & $\mathbf{5 . 1 7}$ & $\mathbf{1 3 . 6 9}$ & $\mathbf{2 2 . 5 0}$ & $\mathbf{2 3 . 9 4}$ \\
\hline
\end{tabular}

Table.6 Mean dry matter plant ${ }^{-1}(\mathrm{mg})$ as affected periodically by different treatments in Fenugreek

\begin{tabular}{|l|c|c|c|c|c|}
\hline \multirow{2}{*}{ Treatment } & \multicolumn{5}{|c|}{ Days after sowing } \\
\cline { 2 - 6 } & $\mathbf{7}$ & $\mathbf{1 4}$ & $\mathbf{2 1}$ & $\mathbf{2 8}$ & At \\
\hline Varieties (V) & & & & \\
\hline V1: Phule Kasturi & 87.75 & 169.99 & 338.93 & 413.98 & $\mathbf{4 7 7 . 0 2}$ \\
\hline V: :MT-1 & 69.12 & 150.48 & 323.11 & 391.20 & $\mathbf{4 5 3 . 1 9}$ \\
\hline V3: Methi Extra Bold & 77.72 & 155.95 & 335.74 & 404.08 & $\mathbf{4 7 0 . 7 3}$ \\
\hline V4: Local variety & 61.38 & 131.22 & 251.87 & 338.07 & $\mathbf{3 6 8 . 3 1}$ \\
\hline S.Em \pm & 0.57 & 1.04 & 0.68 & 0.95 & $\mathbf{1 . 2 3}$ \\
\hline C.D. at 5 \% & 1.98 & 3.60 & 2.36 & 3.29 & $\mathbf{4 . 2 5}$ \\
\hline General mean & $\mathbf{7 4 . 0 0}$ & $\mathbf{1 5 1 . 9 1}$ & $\mathbf{3 1 2 . 4 1}$ & $\mathbf{3 8 6 . 8 3}$ & $\mathbf{4 4 2 . 3 1}$ \\
\hline
\end{tabular}

Table.7 Mean number of days to harvest as influenced by different treatments in fenugreek

\begin{tabular}{|l|c|}
\hline Treatment & Days to harvest \\
\hline Varieties (V) & \\
\hline V1: Phule Kasturi & 37.00 \\
\hline V2: RMT-1 & 32.92 \\
\hline V3: Methi Extra Bold & 34.67 \\
\hline V4: Local variety & 35.17 \\
\hline S.Em \pm & 0.25 \\
\hline C.D. at 5 \% & 0.88 \\
\hline General mean & 34.94 \\
\hline
\end{tabular}


Table.8 Mean weight of fresh foliage plant $^{-1}(\mathrm{mg})$ as affected by different treatments at harvest in fenugreek

\begin{tabular}{|l|c|}
\hline Treatment & Weight of fresh foliage plant ${ }^{-1}(\mathbf{m g})$ \\
\hline Varieties (V) & 1136.21 \\
\hline V1: Phule Kasturi & 958.68 \\
\hline V2: RMT-1 & 1017.74 \\
\hline V3: Methi Extra Bold & 849.47 \\
\hline V4 : Local variety & 1.94 \\
\hline S.Em \pm & 6.73 \\
\hline C.D. at 5 \% & 990.52 \\
\hline General mean & \\
\hline
\end{tabular}

Table.9 Yield contributing character and yield as influenced by different treatments fenugreek

\begin{tabular}{|l|c|}
\hline \multirow{2}{*}{ Treatment } & Fresh foliage yield of fenugreek $\left(\mathrm{q} \mathrm{ha}^{-1}\right)$ \\
\cline { 2 - 2 } & \\
\hline Varieties $(\mathrm{V})$ & 37.67 \\
\hline V1: Phule Kasturi & 31.78 \\
\hline V2: RMT-1 & 33.74 \\
\hline V3: Methi Extra Bold & 28.17 \\
\hline V4 $:$ Local variety & $\mathbf{0 . 0 6}$ \\
\hline S.Em \pm & $\mathbf{0 . 2 2}$ \\
\hline C.D. at 5 \% & 32.84 \\
\hline General mean & \\
\hline
\end{tabular}

Fig.2 Mean plant height $(\mathrm{cm})$ as affected periodically by different varieties in fenugreek

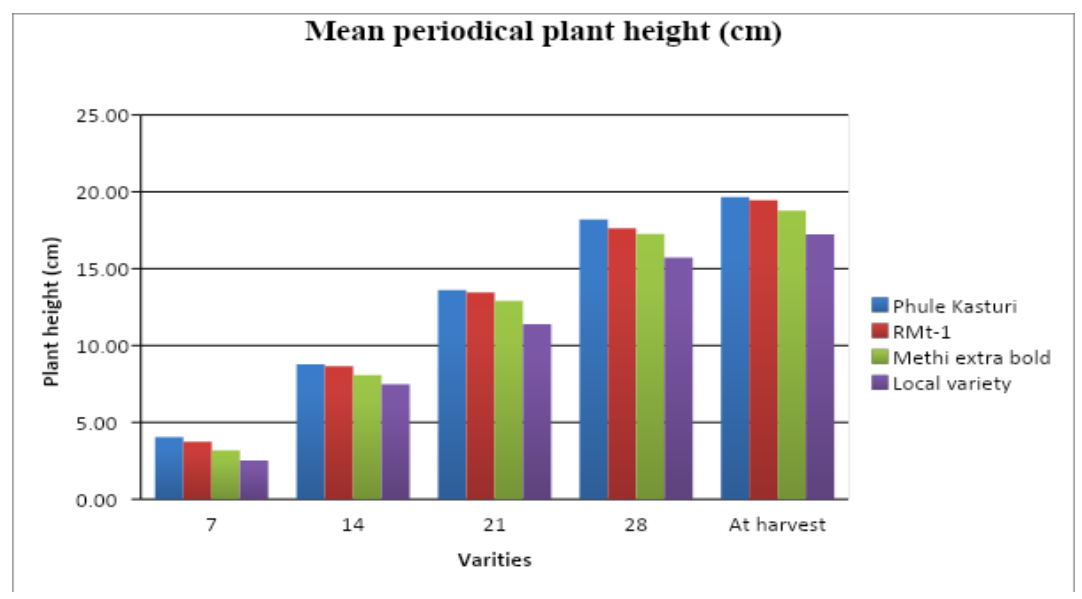


Table.10 Cumulative growing degree days (GDD) as influenced periodically by different varieties and sowing windows in fenugreek

\begin{tabular}{|c|c|c|c|c|c|c|}
\hline \multirow{3}{*}{ Treatment } & & \multicolumn{5}{|c|}{ Cumulative growing degree days $\left({ }^{0}\right.$ Days $)$} \\
\hline & & \multicolumn{5}{|c|}{ Phenological Stage } \\
\hline & & Germination & 2 nodes & 4 nodes & 6 nodes & At harvest \\
\hline \multirow{4}{*}{$\begin{array}{l}\mathrm{V}_{1}: \text { Phule } \\
\text { Kasturi }\end{array}$} & $\begin{array}{l}\mathrm{S}_{1}: 26 \mathrm{MW} \\
\left(25^{\text {th }} \text { June }-1^{\text {st }} \text { July }\right)\end{array}$ & 69.50 & 217.80 & 330.10 & 435.35 & 557.00 \\
\hline & $\begin{array}{l}S_{2}: 27 M W \\
\left(2^{\text {nd }} \text { July }-8^{\text {th }} \text { July }\right)\end{array}$ & 57.65 & 196.85 & 315.70 & 422.45 & 544.65 \\
\hline & $\begin{array}{l}S_{3}: 28 M W \\
\left(9^{\text {th }} \text { July }-15^{\text {th }} \text { July }\right)\end{array}$ & 56.45 & 201.20 & 323.30 & 428.10 & 549.80 \\
\hline & $\begin{array}{l}\mathrm{S}_{4}: 29 \mathrm{MW} \\
\left(16^{\text {th }} \text { July }-22^{\text {nd }} \text { July }\right)\end{array}$ & 62.10 & 224.40 & 352.40 & 466.40 & 589.75 \\
\hline \multirow{4}{*}{$\mathrm{V}_{2}:$ RMT-1 } & $\begin{array}{l}S_{1}: 26 M W \\
\left(25^{\text {th }} \cdot \text { June }-1^{\text {st }} . \text { Julv }\right)\end{array}$ & 51.60 & 190.20 & 315.65 & 404.90 & 496.00 \\
\hline & $\begin{array}{l}S_{2}: 27 M W \\
\left(2^{\text {nd }} \text { July }-8^{\text {th }} \text { July }\right)\end{array}$ & 44.75 & 168.70 & 300.35 & 391.45 & 483.15 \\
\hline & $\begin{array}{l}S_{3}: 28 M W \\
\left(9^{\text {th }} \cdot \text { Iulv }-15^{\text {th }} \cdot \text { Iulv }\right)\end{array}$ & 42.20 & 170.45 & 308.10 & 398.20 & 490.45 \\
\hline & $\begin{array}{l}\mathrm{S}_{4}: 29 \mathrm{MW} \\
\left(16^{\text {th }} \text { July }-22^{\text {nd }} \text { July }\right)\end{array}$ & 46.30 & 190.25 & 335.80 & 435.15 & 528.50 \\
\hline \multirow{4}{*}{$\begin{array}{l}V_{3}: \text { Methi extra } \\
\text { bold }\end{array}$} & $\begin{array}{l}\mathrm{S}_{1}: 26 \mathrm{MW} \\
\left(25^{\text {th }} \text { June }-1^{\text {st }} \text { July }\right)\end{array}$ & 51.60 & 203.70 & 330.10 & 420.25 & 511.80 \\
\hline & $\begin{array}{l}S_{2}: 27 \mathrm{MW} \\
\left(2^{\text {nd }} \text { July }-8^{\text {th }} \text { July }\right)\end{array}$ & 44.75 & 182.60 & 315.70 & 407.25 & 497.35 \\
\hline & $\begin{array}{l}S_{3}: 28 M W \\
\left(9^{\text {th }} \text {.Julv }-15^{\text {th }} \text {.Iulv }\right)\end{array}$ & 42.20 & 185.50 & 323.30 & 412.50 & 520.90 \\
\hline & $\begin{array}{l}\mathrm{S}_{4}: 29 \mathrm{MW} \\
\left(16^{\text {th }} \text { July }-22^{\text {nd }} \text { July }\right)\end{array}$ & 43.30 & 194.60 & 330.40 & 423.20 & $\mathbf{5 2 3 . 5 0}$ \\
\hline \multirow{4}{*}{$\begin{array}{l}\mathrm{V}_{4}: \text { Local } \\
\text { variety }\end{array}$} & $\begin{array}{l}\mathrm{S}_{1}: 26 \mathrm{MW} \\
\left(25^{\text {th }}{\left.\text { June }-1^{\text {st }} \text { July }\right)}\right.\end{array}$ & 69.50 & 217.80 & 315.65 & 420.25 & 527.00 \\
\hline & $\begin{array}{l}S_{2}: 27 \mathrm{MW} \\
\left(2^{\text {nd }} \text { July }-8^{\text {th }} \text { July }\right)\end{array}$ & 57.65 & 196.85 & 300.35 & 407.25 & 546.65 \\
\hline & $\begin{array}{l}S_{3}: 28 M W \\
\left(9^{\text {th }} \text { July }-15^{\text {th }} \text { July }\right)\end{array}$ & 56.45 & 201.20 & 308.10 & 412.50 & 520.90 \\
\hline & $\begin{array}{l}S_{4}: 29 M W \\
(16 \text { July - } 22 \text { July) }\end{array}$ & 62.10 & 224.40 & 335.80 & 423.20 & 535.10 \\
\hline \multicolumn{2}{|l|}{ General mean } & 53.63 & 197.91 & 321.30 & 419.28 & 526.41 \\
\hline
\end{tabular}


Table.11 Light use efficiency (LUE) $\left(\mathrm{g} / \mu\right.$ mole $\left.\mathrm{m}-2 \mathrm{~s}^{-1}\right)$ as affected by different treatments in fenugreek

\begin{tabular}{|c|c|c|c|c|}
\hline $\begin{array}{l}\text { Sr. } \\
\text { No. }\end{array}$ & Treatment & $\begin{array}{l}\text { Cumulative Absorbed } \\
\operatorname{PAR}\left(\mu \text { mole } \mathrm{m}^{-2} \mathrm{~s}^{-1}\right)\end{array}$ & $\begin{array}{l}\text { Dry Matter } \\
\qquad\left(\mathrm{g} / \mathrm{m}^{2}\right)\end{array}$ & $\begin{array}{c}\text { LUE } \\
\left(\mathrm{g} / \mu \text { mole } \mathbf{~ m}^{-2} \mathbf{S}^{-1}\right)\end{array}$ \\
\hline 1 & $\mathrm{~V}_{1} \mathrm{~S}_{1}$ & 212.80 & 158.96 & 0.75 \\
\hline 2 & $\mathrm{~V}_{1} \mathrm{~S}_{2}$ & 218.33 & 158.06 & 0.72 \\
\hline 3 & $\mathrm{~V}_{1} \mathrm{~S}_{3}$ & 216.65 & 158.50 & 0.73 \\
\hline 4 & $\mathrm{~V}_{1} \mathrm{~S}_{4}$ & 218.89 & 157.04 & 0.72 \\
\hline 5 & $\mathrm{~V}_{2} \mathrm{~S}_{1}$ & 226.50 & 154.79 & 0.68 \\
\hline 6 & $\mathrm{~V}_{2} \mathrm{~S}_{2}$ & 228.60 & 149.32 & 0.65 \\
\hline 7 & $\mathrm{~V}_{2} \mathrm{~S}_{3}$ & 227.33 & 149.52 & 0.66 \\
\hline 8 & $\mathrm{~V}_{2} \mathrm{~S}_{4}$ & 233.88 & 147.38 & 0.63 \\
\hline 9 & $\mathrm{~V}_{3} \mathrm{~S}_{1}$ & 221.20 & 156.98 & 0.71 \\
\hline 10 & $\mathrm{~V}_{3} \mathrm{~S}_{2}$ & 224.50 & 156.14 & 0.70 \\
\hline 11 & $\mathrm{~V}_{3} \mathrm{~S}_{3}$ & 222.89 & 156.30 & 0.70 \\
\hline 12 & $\mathrm{~V}_{3} \mathrm{~S}_{4}$ & 225.94 & 154.86 & 0.69 \\
\hline 13 & $\mathrm{~V}_{4} \mathrm{~S}_{1}$ & 233.94 & 123.92 & 0.53 \\
\hline 14 & $\mathrm{~V}_{4} \mathrm{~S}_{2}$ & 242.92 & 122.88 & 0.51 \\
\hline 15 & $\mathrm{~V}_{4} \mathrm{~S}_{3}$ & 235.44 & 123.86 & 0.53 \\
\hline 16 & $\mathrm{~V}_{4} \mathrm{~S}_{4}$ & 245.20 & 117.83 & 0.48 \\
\hline
\end{tabular}

Fig.3 Mean number of functional leaves plant $^{-1}$ as influenced periodically by different varieties in fenugreek

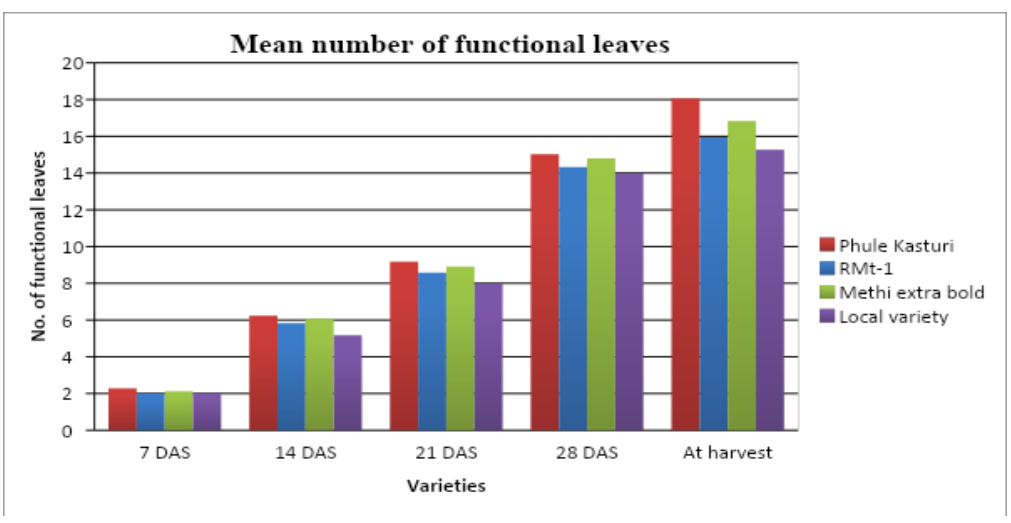


Fig.4 Mean leaf area plant ${ }^{-1}\left(\mathrm{~cm}^{2}\right)$ as affected periodically by different varieties in fenugreek

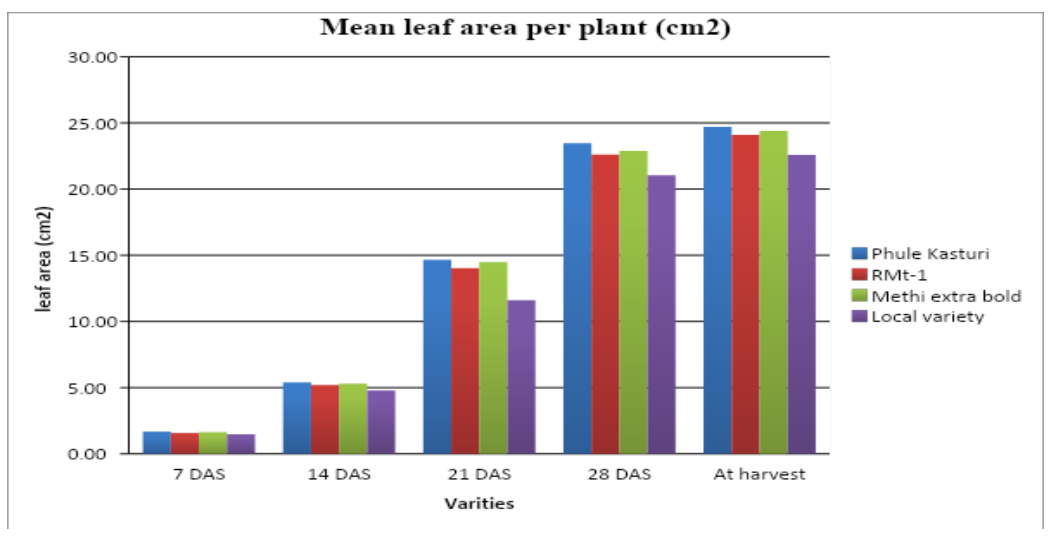

Fig.5 Mean dry matter plant ${ }^{-1}(\mathrm{mg})$ as affected periodically by different varieties in fenugreek

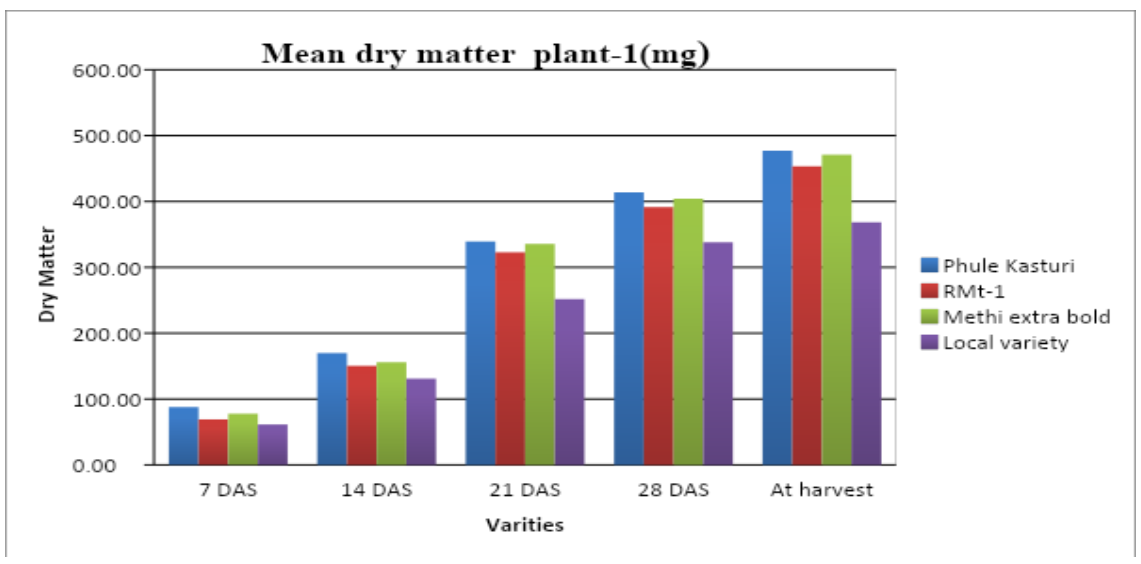

Fig.6 Mean number of days to harvest as affected by different varieties in fenugreek

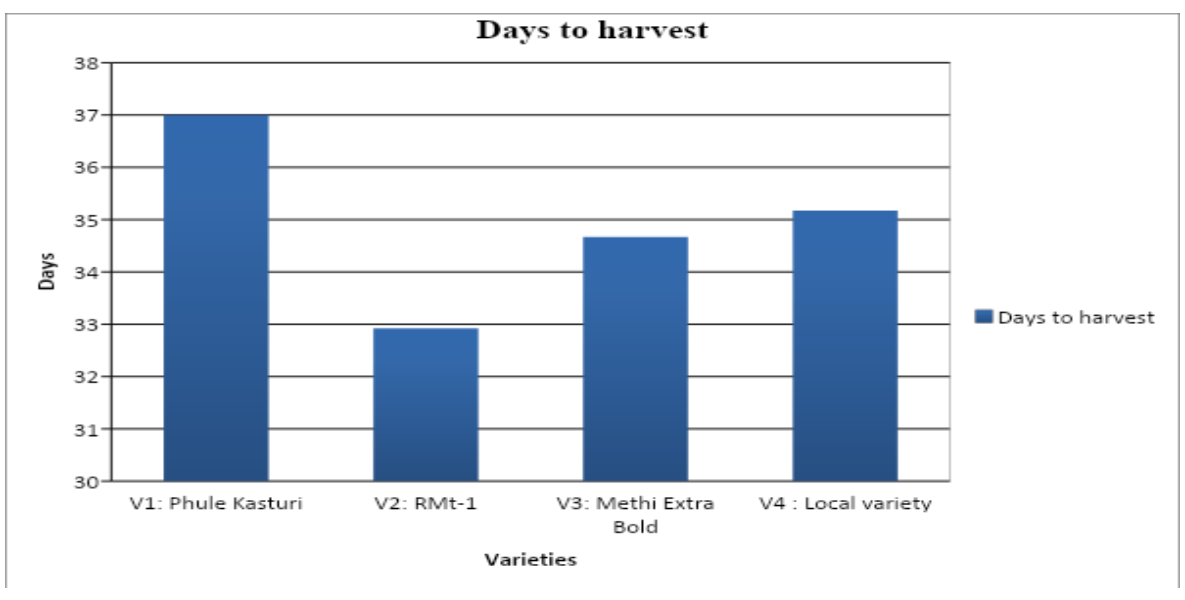


Fig.7 Mean weight of fresh foliage plant ${ }^{-1}(\mathrm{mg})$ as affected by different varieties in Fenugreek

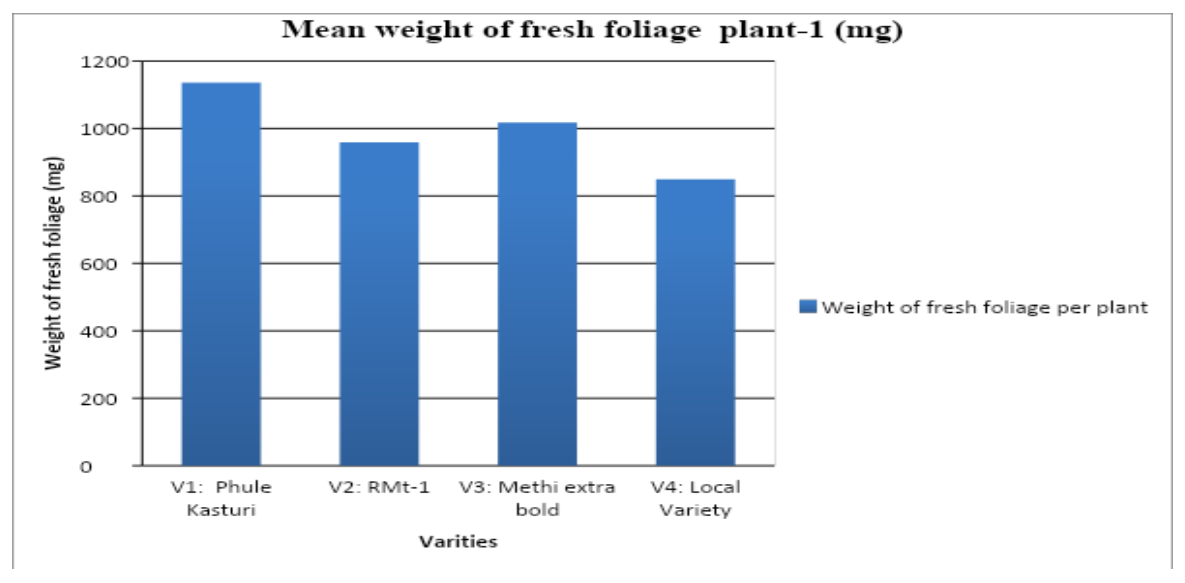

Fig.8 Fresh foliage yield ( $\mathrm{q} \mathrm{ha}^{-1}$ ) as affected by different varieties in fenugreek

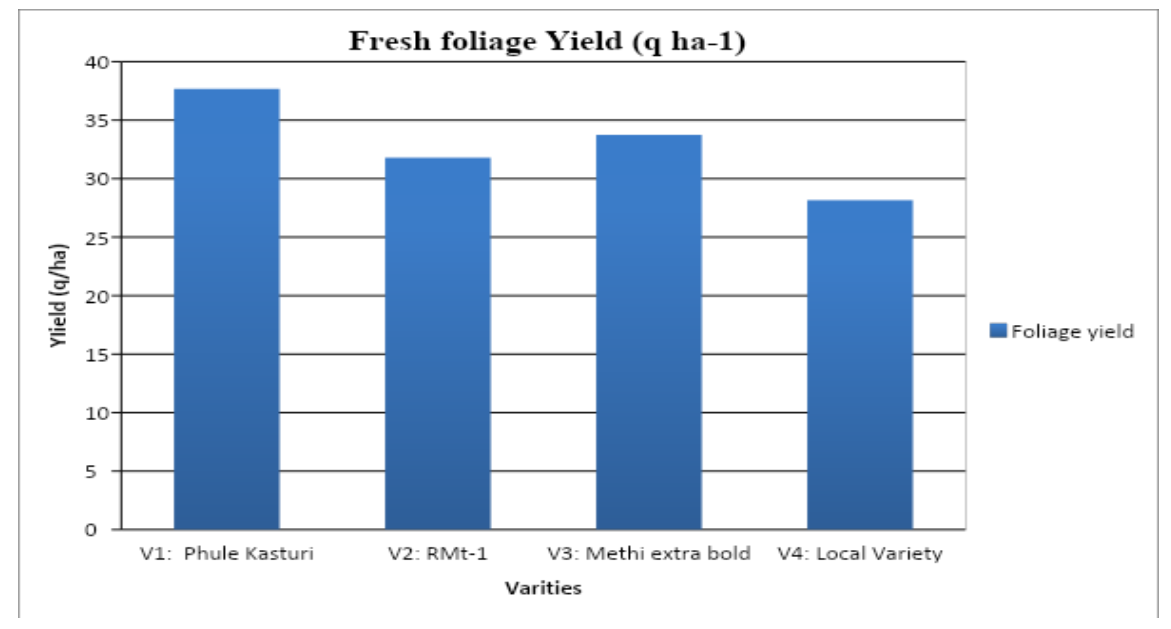

Fig.9 Cumulative growing degree days (GDD) as affected periodically by different varieties in fenugreek

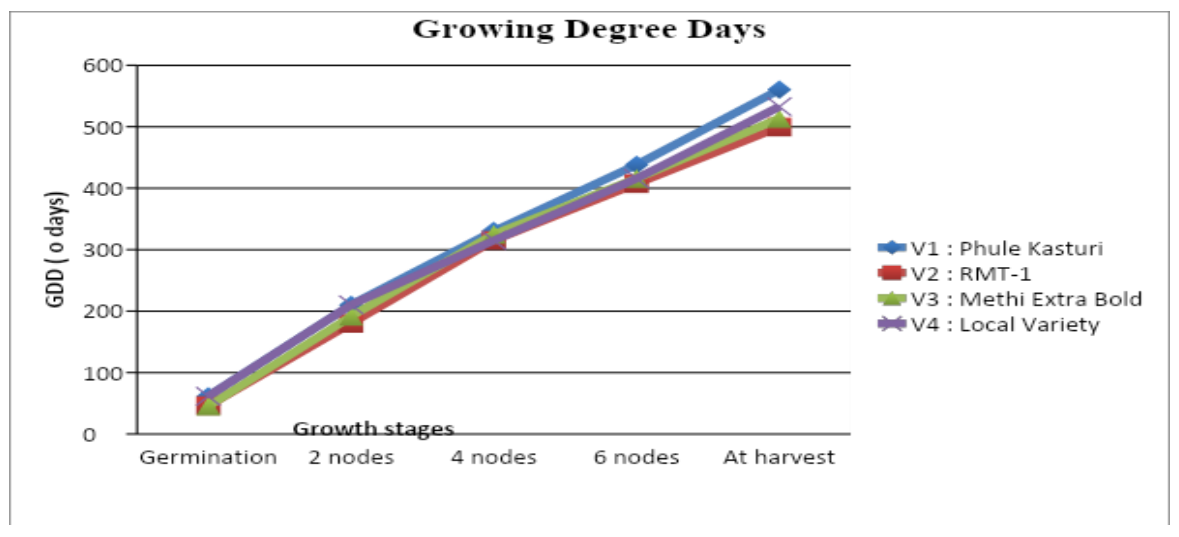


Fig.10 Light Use Efficiency (LUE) (g/ $/ \mu$ mole $\left.m-2 s^{-1}\right)$ as affected by different treatments in fenugreek

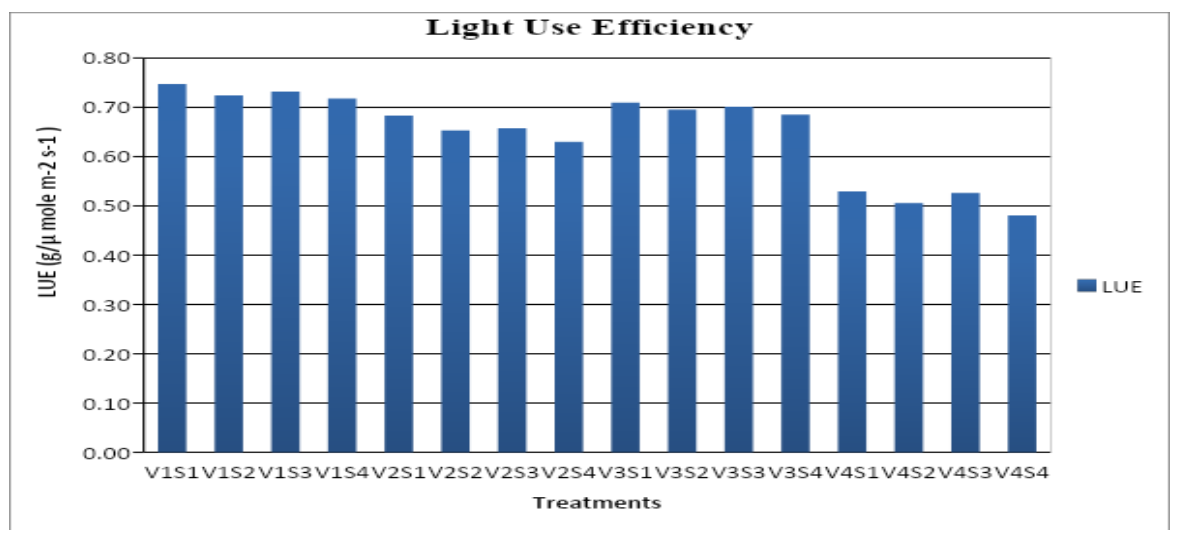

\section{Days to harvest}

The data regarding mean days to harvest influenced by different fenugreek varieties and sowing windows are presented in Table 7 and graphically depicted in Fig 6. The days to harvest were significantly influenced due to varieties and sowing windows. The mean days to harvest was 34.94 .

The data regarding mean days to harvest as influenced by different fenugreek varieties are presented in Table 7. It was significantly influenced due to varieties.

A variety RMT-1 recorded minimum days to harvest (32.92) and maximum for variety Phule Kasturi (37.00). This was due to the climatic conditions at that windows and genetic factors of those varieties. Revanappa et al., (2012), Aggrwal et al., (2013), Singh et al., (2013), Sharma (2015), Girdhar et al., (2016) and Latye et al., (2016) reported similar findings.

\section{Post harvest observations}

\section{Weight of fresh foliage plant ${ }^{-1}$ at harvest}

The data recorded on weight of fresh foliage plant $^{-1}(\mathrm{mg})$ in fenugreek as affected by different treatments are presented in Table 8 and graphically represented in Fig.7.

The weight of fresh foliage plant ${ }^{-1}$ with respect to different fenugreek varieties showed significant results (Table 8). The highest weight of fresh foliage plant $^{-1}$ $(1136.21 \mathrm{mg})$ recorded with variety Phule Kasturi over other varieties. The next varieties in order of sequence were Methi Extra Bold (1017.74 mg), RMT-1 (958.68 mg) and Local variety $(849.47 \mathrm{mg})$.

This might be due to varietal characters. Revanappa et al., (2012), Aggrwal et al., (2013), Singh et al., (2013), Sharma (2015), Girdhar et al., (2016) and Latye et al., (2016) reported similar findings.

\section{Yield}

The data pertaining to fresh foliage yield plant $^{-1}(\mathrm{mg})$ and foliage yield $\left(\mathrm{q} \mathrm{ha} \mathrm{ha}^{-1}\right)$ in fenugreek as affected by different treatments are presented in Table 9 and depicted in Fig.8.

\section{Foliage yield}

Significant and progressive difference was observed in foliage yield of fenugreek due to different varieties and windows of sowing. It produced mean fresh foliage yield of $32.84 \mathrm{q}$ $\mathrm{ha}^{-1}$. 
The varieties of fenugreek influenced significantly to foliage yield $\left(\mathrm{q} \mathrm{ha}^{-1}\right)$. A variety Phule Kasturi produced higher yield $\left(37.67 \mathrm{q} \mathrm{ha}^{-1}\right)$ than other varieties in order of yield sequence are Methi Extra Bold (33.74 q $\left.\mathrm{ha}^{-1}\right)$, RMT-1(31.78 $\left.\mathrm{q} \mathrm{ha}^{-1}\right)$ and Local variety $\left(28.17 \mathrm{q} \mathrm{ha}^{-1}\right.$ ) foliage yield (Table 9). A variety Methi Extra Bold recorded better results than other due to its genetical characters and favourable climatic conditions. Revanappa et al., (2012), Aggrwal et al., (2013), Singh et al., (2013), Sharma (2015), Girdhar et al., (2016) and Latye et al.(2016) reported similar findings.

\section{Meteorological observations}

\section{Growing Degree Days (GDD)}

The growing degree days were calculated by using daily maximum, minimum and base temperatures for kharif fenugreek and presented in Table 10 and graphically depicted in fig.9.

Among the all varieties, Phule Kasturi induced more heat load (560.30 heat units) than rest of the fenugreek varieties because of its longer duration, whereas, variety RMT-1 showed the lowest heat load (i.e. 499.53 heat units) because of its shorter growth period (Table 10). Similar findings were reported by Bagade et al., (2017) and Ghodake (2019).

\section{Light use efficiency by fenugreek crop canopy}

The data pertaining light use efficiency by fenugreek crop canopy as affected by different treatments are presented in Table 11 and depicted in Fig.10.

Among the all varieties, Phule Kasturi indicated more light use efficiency i.e. $0.73 \mathrm{~g}$ $/ \mu$ mole $\mathrm{m}-2 \mathrm{~s}^{-1}$ than rest of the varieties (Table 11), whereas, local variety showed the lowest LUE i.e. $0.51 \mathrm{~g} / \mu$ mole $\mathrm{m}-2 \mathrm{~s}^{-1}$. Similar findings were reported by Bagade et al., (2017)

On the basis of the field experimentation for a season, it could be concluded that: Among the four varieties of fenugreek, Phule Kasturi variety produced significantly higher yield $\left(37.67 \mathrm{q} \mathrm{ha}^{-1}\right.$ ) over Methi Extra Bold (33.74 q $\left.\mathrm{ha}^{-1}\right)$, RMT-1 (31.78 q ha ${ }^{-1}$ ) and Local variety $\left(28.17 \mathrm{q} \mathrm{ha}^{-1}\right)$.

However, above conclusions are based on one season study. For confirmation of these results, the investigation needs to be repeated.

\section{References}

Aggrwal, K.B., Ranjan,J.K., Rathore,S.S., Saxena., S.N. and Mishra,B.K. 2013. Changes in physical and biochemical properties of fenugreek (Trigonella $s p$. L.) leaf during different growth stages. Internat. J. Seed Spices. 3(1): 31-35.

Anonymous, 2012. U.S. Department of Health and Human Services 2012. National Institutes of Health Web site: <www.ncbi.nlm.nih.gov/sites/entre>.

Bagade,S.V., S.B.Kharbade and A.A.Shaikh.2017. Impact of sowing windows on different yield components and yield of soybean under changing climatic conditions at Pune. Trends in Biosci.10(10): $1891^{-1} 893$.

Flammang, A.M., Cifone, M.A., Erexson, G.L. and Stankowski, L.F. 2004. Genotoxicity testing of a fenugreek extract. Food and Chemical Toxicol. 42:1769-1775.

Ghodake, S. V. 2019. Response of black gram (Vigna mungo (L.) Hepper) varieties under different sowing windows in kharif season. M.Sc. (Agri.) thesis submitted to MPKV, Rahuri.

Giridhar,K., Surya Kumari,S., Rajani,A., 
Sarada, C. and Naram Naidu L. 2016. Identification of potential genotypes of fenugreek in rainfed vertisols for yield and diosgenin content. Indian J.Agric.Res.(50):311-317.

Govindaraj, K., Balakrishnan, S., Shoba,N. and Somasundaram, E. 2019. Influence of growing environment on growth and yield of fenugreek leaves under shade net and open condition. Internat.J.Chemical Studies. 7(3): 21022105.

Latye, P.T., Bharad, S.G., Kale, V.S., Nandeshwar, V.N. and Kholia, A. 2016. Varietal performance of fenugreek under Akola conditions. Internat.J.Minor Fruits, Medicinal and Aromatic Pl. 2(1): 32 - 34.

Madar, Z., Stark, A.H. 2002. New legume sources as therapeutic agents. Brit.J. Nutr., 88, S287- S292.

Mehta, R.S., Patel, B.S., Singh, R. K., Meena, S.S. and Malhotra, S. K. 2013. Growth and yield of fenugreek (Trigonella foenum-graecum L.) as influenced by irrigation levels and weed management practices. J.Spices and Aromatic Crops. 19:14-22.

Panse, V. G. and Sukhatme, P. V. 1985. Statistical Methods for Agricultural Workers. Indian Council of Agricultural Research Publication, 87-89.

Revanappa, S.B., Kamannavar, P.Y., Vijaykumar, A.G., Ganajaxi, M., Arunkumar, B. and Salimath,

Sharma, O.P.2015. Performance of black gram varieties to varying row spacing under Agro-climatic conditions of Vindhyan plateau. M.Sc. (Agri.) Thesis submitted to RVSKVV, Gwalior.

Singh, S.P., Sandhu, S.K., Dhahiwal, L.K. and Singh, I. 2013. Effect of Planting Geometry on Microclimate, Growth and Yield of Mungbean (Vigna radiata $\mathrm{L}$.). J.Agril. Physics. 12(1): 70-73.

\section{How to cite this article:}

Supriya K. Ghadge, A. A. Shaikh, J. D. Jadhav, V. A. Sthool, A. B. Bhosale and Bagade, S. V. 2021. Performance of Fenugreek (Trigonella foenum-graecum L.) Varieties for Table Purpose under kharif Season. Int.J.Curr.Microbiol.App.Sci. 10(02): 2408-2421. doi: https://doi.org/10.20546/ijcmas.2021.1002.286 